\title{
Utilisation optimale des cabinets automatisés décentralisés : le point sur les risques de détournement des substances désignées
}

\author{
Sophie Dubois, Emmy Bernier, Julie Rivard, Lavina Yu, Suzanne Atkinson et Jean-François Bussières
}

\section{INTRODUCTION}

T e circuit du médicament en établissement de santé est _complexe et comporte au moins 54 étapes $^{1}$. Afin de l'optimiser, l'implantation de meilleures pratiques organisationnelles et le recours à différents types de technologies sont possibles $^{2}$. Les cabinets automatisés décentralisés (CAD) font partie de ces technologies depuis au moins deux décennies. Cependant, on ne compte qu'une cinquantaine d'articles sur le sujet en utilisant l'expression <automated dispensing cabinets> dans Pubmed. Brisseau et coll. se sont intéressés à la conformité des CAD de type Acudose (renommé Aesynt puis Omnicell depuis l'étude) au Canada en se basant sur les lignes directrices de l'Institute for Safe Medication Practices publiées en 2008 3,4. Ces travaux mettent en évidence certaines lacunes des CAD en dépit de la publication de lignes directrices. Les CAD comportent des avantages et des inconvénients 5 . Les avantages sont notamment la possibilité d'interfacer les CAD avec le logiciel du système d'information de la pharmacie, ce qui permet au personnel soignant d'accéder à la liste de médicaments prescrits à un patient donné. De plus, l'accès aux médicaments est sécurisé par biométrie ou par code d'accès individualisé, ce qui assure la traçabilité des transactions de chaque usager. Les CAD sont notamment utilisés pour la surveillance des substances à potentiel d'abus, soit les narcotiques (p. ex. morphine), les drogues contrôlées (p. ex. phénobarbital) et les benzodiazépines (p. ex. midazolam) ${ }^{6}$. Différents rapports de surveillance sont disponibles grâce au logiciel de gestion des CAD (Connect-Rx) et permettent de suivre les accès ainsi que l'utilisation des médicaments et d'évaluer la conformité des pratiques. Les inconvénients des CAD sont notamment leur coût relativement élevé, soit des dizaines de milliers de dollars par CAD. Ils ne permettent pas de prévenir tous les risques liés à l'utilisation des médicaments, et les mécanismes de sécurité peuvent être contournés à des fins de vol ou d'utilisation inappropriée.
L'accès fréquent des professionnels et d'autres intervenants du réseau de la santé aux substances désignées, dans le cadre de leur pratique, facilite leur détournement pour une utilisation personnelle ou pour en faire le trafic. Il est reconnu que 10 à $15 \%$ des professionnels de la santé abusent de médicaments ou d'autres substances illicites. Ce taux est similaire à celui observé dans la population générale ${ }^{7}$. Quelques articles décrivent la prévalence d'abus de médicaments parmi les professionnels de la santée-11.

Le personnel soignant est conscient des obligations juridiques en matière de surveillance de l'entreposage des substances désignées et des conséquences en cas de détournement, pouvant inclure notamment, amende, radiation disciplinaire ou suspension de travail temporaire ou définitive. En consultant la principale bibliothèque virtuelle d'information juridique canadienne, nous avons trouvé huit jugements portant sur le détournement de substances désignées à l'aide d'un CAD, impliquant sept infirmières et un pharmacien ${ }^{12}$. Le pharmacien impliqué dans le détournement s'est approprié 20 fioles d'hydromorphone sur une période de 10 jours dans un CAD des urgences d'un centre hospitalier universitaire, en retirant le médicament au nom de patients qui ne devaient pas en recevoir. Ce type d'opérationne génère aucun écart s'il est complété sans erreur. Le pharmacien a fait l'objet d'une radiation de 18 mois de son ordre professionnel. Le personnel infirmier recueille l'information sur les détournements de substances désignées, ceux-ci sont toutefois plus rarement associés aux pharmaciens hospitaliers. Ce cas a contribué à sensibiliser les chefs de départements de pharmacie à la révision des mesures de surveillance des substances désignées au sein de leur département. En 2015, Brenn et coll. ont publié une étude décrivant l'intégration de différents outils afin d'assurer une meilleure surveillance de la dispensation et de l'utilisation des substances désignées en établissement de santé ${ }^{13}$. Nous nous sommes inspirés de ces travaux pour revoir les modalités de surveillance 
de l'utilisation des substances désignées, dispensées par CAD au sein de notre établissement.

L'objectif principal de cette étude est d'établir un profil des écarts lors du dénombrement des substances désignées dispensées par CAD. L'objectif secondaire est de déterminer les modes de défaillance des CAD permettant le détournement des substances désignées et de proposer des mesures supplémentaires de surveillance.

\section{MÉTHODE}

L'étude descriptive et rétrospective s'est déroulée au Centre hospitalier universitaire (CHU) Sainte-Justine. Dix-neuf CAD de type Acudose (renommé Aesynt puis Omnicell depuis leur achat) sont implantés depuis 2009 au sein des unités d'oncologie $(n=4)$, de néonatologie $(n=3)$, de chirurgie $(n=3)$, de pédiatrie $(n=3)$, de soins de longue durée $(n=2)$, des soins intensifs pédiatriques $(n=2)$ et des urgences $(n=2)$. Les CAD sont utilisés pour la dispensation de la presque totalité des doses de substances désignées et pour les premières doses des autres médicaments. Le personnel technique de la pharmacie, sous la supervision d'un pharmacien gestionnaire, assure le remplissage quotidien des $\mathrm{CAD}$ et la gestion des écarts en collaboration avec le personnel infirmier. En principe, un administrateur doit résoudre tout écart et l'inscrire au CAD. Des relances sont effectuées par le personnel de la pharmacie jusqu'à la résolution de l'écart. Une politique et procédure balise l'ensemble de ces activités. Toute opération réalisée dans un CAD est transmise au système d'information de la pharmacie. Le personnel de la pharmacie peut constater en temps réel les sorties de médicament effectuées par le personnel soignant. Toutefois, cela ne permet pas la détection d'un détournement de dose.

Le suivi des substances désignées dans les CAD repose prioritairement sur un système obligatoire de dénombrement en aveugle par l'usager, paramétré dans le logiciel de gestion des $\mathrm{CAD}$. Dès l'ouverture d'une pochette contenant une substance désignée, l'usager doit inscrire le dénombrement du contenu de la pochette sans connaître la dernière quantité enregistrée par le CAD. Le CAD concilie cette donnée avec l'information de la dernière transaction mémorisée. Dans le cas où les informations saisies ne concordent pas, un écart se crée au CAD et doit être résolu par un administrateur du système. Un écart peut se créer lorsque la quantité réelle sortie est différente de celle que l'usager inscrit au CAD. Ce type d'écart demande qu'un administrateur fasse une recherche, parfois complexe, et qu'il

\section{Tableau 1. Profil des mouvements et des écarts d'inventaire par cabinet automatisé décentralisé} du 3 juin au 2 novembre 2015

\begin{tabular}{|c|c|c|c|c|}
\hline \multirow{2}{*}{$\begin{array}{l}\text { Cabinet } \\
\text { automatisé } \\
\text { décentralisé }\end{array}$} & \multirow{2}{*}{$\begin{array}{c}\text { Nombre }(\%) \\
\text { de transactions } \\
(n=17950)\end{array}$} & \multicolumn{2}{|c|}{ Écarts d'inventaire totaux } & \multirow{2}{*}{$\begin{array}{c}\text { Nombre }(\%) \text { d'écarts } \\
\text { d'inventaire complexes } \\
(n=91)\end{array}$} \\
\hline & & $\begin{array}{c}\text { Nombre }(\%) \\
(n=221)\end{array}$ & $\begin{array}{l}\text { Taux d'écart par } \\
\text { transaction (\%) }\end{array}$ & \\
\hline \multicolumn{5}{|l|}{ Oncologie } \\
\hline $1 \mathrm{~A}$ & $837 \quad(4,7)$ & $6(2,7)$ & 0,7 & $4(4,4)$ \\
\hline 1B & $372(2,1)$ & $5(2,3)$ & 1,3 & $3(3,3)$ \\
\hline 2 & $868(4,8)$ & $14(6,3)$ & 1,6 & $5(5,5)$ \\
\hline 3 & $639(3,6)$ & $4(1,8)$ & 0,6 & $2(2,2)$ \\
\hline \multicolumn{5}{|c|}{ Néonatologie } \\
\hline 1 & $108(0,6)$ & $3(1,4)$ & 2,8 & $2(2,2)$ \\
\hline 2 & $213(1,2)$ & $2(0,9)$ & 0,9 & $2(2,2)$ \\
\hline 3 & $2581(14,4)$ & $44(19,9)$ & 1,7 & $8(8,8)$ \\
\hline \multicolumn{5}{|l|}{ Chirurgie } \\
\hline 1 & $33(0,2)$ & $0(0,0)$ & 0,0 & $0(0,0)$ \\
\hline 2 & $3525(19,6)$ & $28(12,7)$ & 0,8 & $19(20,9)$ \\
\hline 3 & $453(2,5)$ & $3(1,4)$ & 0,7 & $2(2,2)$ \\
\hline \multicolumn{5}{|l|}{ Pédiatrie } \\
\hline 1 & $242(1,3)$ & $4(1,8)$ & 1,7 & $1(1,1)$ \\
\hline 2 & $375(2,1)$ & $5(2,3)$ & 1,3 & $3(3,3)$ \\
\hline 3 & $285(1,6)$ & $3(1,4)$ & 1,1 & $0(0,0)$ \\
\hline \multicolumn{5}{|c|}{ Soins longue durée } \\
\hline 1 & $495 \quad(2,8)$ & $13(5,9)$ & 2,6 & $4(4,4)$ \\
\hline 2 & $27 \quad(0,2)$ & $7 \quad(3,2)$ & 25,9 & $4(4,4)$ \\
\hline \multicolumn{5}{|c|}{ Soins intensifs } \\
\hline 1 & $3620(20,2)$ & $38(17,2)$ & 1,0 & $16(17,6)$ \\
\hline 2 & $1016 \quad(5,7)$ & $16(7,2)$ & 1,6 & $4 \quad(4,4)$ \\
\hline \multicolumn{5}{|l|}{ Urgences } \\
\hline 1 & $1923(10,7)$ & $20(9,0)$ & 1,0 & $11(12,1)$ \\
\hline 2 & $338 \quad(1,9)$ & $6(2,7)$ & 1,8 & $1(1,1)$ \\
\hline Total & $17950(100)$ & $221(100)$ & 1,2 & $91(100)$ \\
\hline
\end{tabular}


Tableau 2. Profil du nombre d'écarts d'inventaire par quart de travail des infirmières du 3 juin au 2 novembre 2015.

\begin{tabular}{lccc} 
Quart de travail & $\begin{array}{c}\text { Nombre }(\%) \text { de } \\
\text { transactions totales } \\
(\boldsymbol{n}=\mathbf{1 7} \text { 950) }\end{array}$ & $\begin{array}{c}\text { Nombre }(\%) \\
\text { d'écarts totaux } \\
(\boldsymbol{n}=\mathbf{2 2 1})\end{array}$ & $\begin{array}{c}\text { Taux d'écart } \\
\text { par } \\
\text { transaction (\%) }\end{array}$ \\
\hline Quart de jour $(7 \mathrm{~h}-15 \mathrm{~h})$ & $8314(46,3)$ & $98(44,3)$ & 1,2 \\
Quart de soir $(15 \mathrm{~h}-23 \mathrm{~h})$ & $5648(31,5)$ & $78(35,3)$ & 1,4 \\
Quart de nuit $(23 \mathrm{~h}-7 \mathrm{~h})$ & $3988(22,2)$ & $45(20,4)$ & 1,1 \\
\hline
\end{tabular}

\section{Tableau 3. Profil du nombre de mouvements et d'écarts d'inventaire par substance désignée du 3 juin} au 2 novembre 2015

\begin{tabular}{|c|c|c|c|c|c|}
\hline \multirow{2}{*}{$\begin{array}{l}\text { Classe thérapeutique } \\
\text { Amphétamine }\end{array}$} & \multirow{2}{*}{$\begin{array}{l}\text { Médicament } \\
\text { Méthylphénidate - voie orale }\end{array}$} & \multicolumn{2}{|c|}{$\begin{array}{c}\text { Nombre }(\%) \text { de } \\
\text { transactions totales } \\
(n=17950)\end{array}$} & \multicolumn{2}{|c|}{$\begin{array}{c}\text { Nombre (\%) } \\
\text { d'écarts totaux } \\
(n=221)\end{array}$} \\
\hline & & 365 & $(2,03)$ & 8 & $(3,62)$ \\
\hline Autre anesthésique & Kétamine - voie injectable & 1379 & $(7,68)$ & 12 & $(5,43)$ \\
\hline Autre hypnotique & Étomidate - voie injectable & 50 & $(0,28)$ & 0 & $(0)$ \\
\hline \multirow[t]{4}{*}{ Barbituriques } & Pentobarbital - voie injectable & 5 & $(0,03)$ & 0 & (0) \\
\hline & Phénobarbital - voie injectable & 162 & $(0,90)$ & 2 & $(0,90)$ \\
\hline & Phénobarbital - voie orale & 43 & $(0,24)$ & 7 & $(3,17)$ \\
\hline & Total barbituriques & 210 & $(1,17)$ & 9 & $(4,07)$ \\
\hline \multirow[t]{9}{*}{ Benzodiazépines } & Clobazam - voie orale & 54 & $(0,30)$ & 2 & $(0,90)$ \\
\hline & Clonazepam - voie orale & 11 & $(0,06)$ & 0 & (0) \\
\hline & Diazepam - voie orale & 140 & $(0,78)$ & 2 & $(0,90)$ \\
\hline & Diazepam - voie injectable & 12 & $(0,07)$ & 0 & (0) \\
\hline & Lorazepam - voie orale & 375 & $(2,09)$ & 8 & $(3,62)$ \\
\hline & Lorazepam - voie injectable & 778 & $(4,33)$ & 14 & $(6,33)$ \\
\hline & Midazolam - voie injectable & 739 & $(4,12)$ & 10 & $(4,52)$ \\
\hline & Nitrazepam - voie orale & 6 & $(0,03)$ & 1 & $(0,45)$ \\
\hline & Total benzodiazépines & 2115 & $(11,78)$ & 37 & $(16,74)$ \\
\hline Cannabinoïde & Nabilone - voie orale & 147 & $(0,82)$ & 2 & $(0,90)$ \\
\hline \multirow[t]{10}{*}{ Opiacés } & Acétaminophène + codéine - voie orale & 15 & $(0,08)$ & 0 & $(0)$ \\
\hline & Codéine - voie orale & 7 & $(0,04)$ & 0 & (0) \\
\hline & Codéine - voie injectable & 3 & $(0,02)$ & 0 & (0) \\
\hline & Fentanyl - voie injectable & 3293 & $(18,35)$ & 45 & $(20,36)$ \\
\hline & Hydromorphone - voie orale & 884 & $(4,92)$ & 7 & $(3,17)$ \\
\hline & Hydromorphone - voie injectable & 1631 & $(9,09)$ & 21 & $(9,50)$ \\
\hline & Mépéridine - voie injectable & 3 & $(0,02)$ & 0 & (0) \\
\hline & Morphine - voie orale & 586 & $(3,26)$ & 4 & $(1,81)$ \\
\hline & Morphine - voie injectable & 7262 & $(40,46)$ & 76 & $(34,39)$ \\
\hline & Total opiacés & 13684 & $(76,23)$ & 153 & $(69,23)$ \\
\hline
\end{tabular}

parvienne à justifier l'absence des produits manquants. Ce type d'écart est décrit ici comme un écart complexe. Un écart peut se produire si l'usager inscrit la mauvaise quantité au dénombrement, soit en raison d'une erreur de comptage ou d'une erreur de frappe. Dans cette dernière situation, le dénombrement devrait être remis à jour lors de la transaction suivante, ce qui crée naturellement un deuxième écart dans le sens inverse du premier, qui annule en quelque sorte l'erreur du premier usager. Ce type d'écart demande une justification, mais puisqu'il n'y a en réalité aucun produit manquant, la résolution de ces situations est généralement simple. Ce type d'écart est décrit ici comme un écart simple.

Afin d'établir le profil des écarts d'inventaire de substances désignées dispensées par CAD, nous avons extrait tous les écarts d'inventaire recueillis par le système de gestion des CAD (Datastation ${ }^{\circledR}$ et Connect Rx®, Pittsburgh, PA, ÉUA) entre le 3 juin et le 2 novembre 2015. Nous avons calculé le taux d'écart d'inventaire par transaction et établi un profil descriptif de ces écarts par unité de soins et CAD, par heure, par quart de travail et par dénomination commune de médicament. Afin de décrire le profil des écarts d'inventaire, nous avons pris en considération les écarts totaux (c.-à-d. écarts simples et écarts complexes) et les écarts complexes. Le calcul des coefficients de corrélation (Pearson) entre le nombre de transactions et le nombre d'écarts d'inventaire a été réalisé selon différents critères : par unité de soins et par CAD, par heure, par quart de travail et par substance désignée. Il a débouché sur la production de statistiques descriptives. 
Tableau 4. Profil des modes de défaillance décelés par le panel d'experts et le groupe de discussion pour chaque activité de gestion des cabinets automatisés décentralisés (CAD)

Activités

Modes de défaillance (Mots-clés et description)
Usagers concernés et rapports de surveillance* concernés

- Assistant technique en pharmacie - Refill activities*
Reapprovisionnement les CAD 1. Erreur de dénombrement - Dénombrement visuel inexact du nombre •
d'unités d'un médicament présent dans la pochette par rapport à
la quantité réelle

2. Erreur de saisie - Saisie inexacte du nombre d'unités d'un médicament par rapport au dénombrement ou à l'ajout au CAD

3. Erreur de remplissage - Ajout d'une quantité inexacte d'un médicament par rapport à la quantité saisie au CAD ou ajout d'un autre médicament que celui saisi au CAD

4. Erreur de gestion d'un retour - Remise d'un médicament dans la mauvaise pochette d'un CAD après la gestion du retour

5. Vol après sortie du stock de la pharmacie - Conservation du médicament après la sortie de la pharmacie et avant le réapprovisionnement du CAD

6. Vol malgré la lecture par code-barres - En dépit d'une lecture de code-barres d'un médicament à ajouter, conservation du médicament

7. Vol dans le chariot de transport - Vol d'un médicament dans le chariot de transport laissé sans surveillance avant le réapprovisionnement du CAD

8. Vol dans le bac de retours - Vol d'un médicament dans le bac des retours du CAD

9. Vol par substitution du médicament par un autre médicament Remplacement du contenu d'un médicament par une autre substance (p. ex. retrait d'un opiacé d'une fiole et remplacement par un soluté) avant le réapprovisionnement du CAD et conservation du médicament

II. Sortie de médicaments dans les $C A D$

III. Gestion des écarts d'inventaire au CAD
10. Opération faite avec l'identifiant d'une tierce personne Un usager peut faire des opérations dans le CAD au nom d'une autre personne dans les situations suivantes : vol d'identifiant, partage d'identifiant, session de l'usager précédent restée ouverte

11. Retrait de médicament sans administration au patient L'intervenant retire un médicament du CAD sans l'administrer au patient et le conserve. L'intervenant peut également décrire l'administration du médicament sur une feuille d'administration papier ou électronique des médicaments, même si la dose n'est pas administrée

12. Retrait d'une quantité de médicament supérieure à ce qui est nécessaire - L'intervenant retire du CAD une quantité de médicament supérieure à ce qui est nécessaire pour l'administration au patient et conserve l'excédent

13. Retrait d'un médicament pour le patient d'un autre intervenant L'intervenant A sort le médicament pour un patient de l'intervenant B et conserve le médicament

14. Substitution d'un médicament par une autre substance L'intervenant remplace le médicament par une autre substance avant l'administration au patient et le conserve

15. Sortie d'un médicament en mode hors profil - L'intervenant sort le médicament en mode hors profil sans que le médicament soit prescrit pour un patient donné et le conserve

16. Sortie d'un médicament à l'avance - L'intervenant sort un médicament du CAD avant l'heure d'administration pour gagner du temps. Le médicament peut être volé ou perdu s'il n'est pas mis en lieu sûr

17. Confusion entre les unités de mesure lors des mouvements L'intervenant saisit l'information en $\mathrm{ml}$ au lieu de mg ou de quantité. Cette confusion peut engendrer un écart d'inventaire et contribuer au risque de vol s'il reste irrésolu

18. Modification volontaire des décomptes - L'intervenant peut prendre • Tous une quantité de médicaments supérieure à ce qui est nécessaire, la conserver, puis modifier le dénombrement de deux façons : a. Lors du dénombrement, mettre un nombre inférieur à la quantité réellement présente dans la pochette ce qui crée un écart d'inventaire par rapport à la transaction précédente

b. Lors de la saisie de la quantité prélevée, mettre un nombre inférieur à la quantité réellement prise et création d'un écart d'inventaire lors de la transaction suivante
- Tous les intervenants ayant accès aux CAD

- Controlled substance dispense*
- Discrepancy by station* 
Tableau 4. Profil des modes de défaillance décelés par le panel d'experts et le groupe de discussion pour chaque activité de gestion des cabinets automatisés décentralisés (CAD) (suite et fin)

\begin{tabular}{|c|c|c|}
\hline Activités & $\begin{array}{l}\text { Modes de défaillance } \\
\text { (Mots-clés et description) }\end{array}$ & $\begin{array}{l}\text { Usagers concernés et } \\
\text { rapports de surveillance* } \\
\text { concernés }\end{array}$ \\
\hline \multirow[t]{4}{*}{$\begin{array}{l}\text { IV. Retours et rebuts de } \\
\text { médicaments }\end{array}$} & $\begin{array}{l}\text { 19. Retour non effectué au cabinet - Le médicament sorti n'est pas } \\
\text { utilisé par le patient, et l'intervenant le conserve au lieu d'enregistrer } \\
\text { un retour }\end{array}$ & $\begin{array}{l}\text { - Tous } \\
\text { - Return bin activities* }\end{array}$ \\
\hline & $\begin{array}{l}\text { 20. Retour fait au cabinet sans dépôt du produit - L'intervenant inscrit } \\
\text { le retour du médicament au CAD sans le déposer dans le bac de retour } \\
\text { et le conserve }\end{array}$ & \\
\hline & $\begin{array}{l}\text { 21. Absence de d'inscription des rebuts - Le médicament sorti n'est } \\
\text { utilisé qu'en partie pour le patient; l'intervenant conserve l'excédent } \\
\text { au lieu de l'inscrire devant témoin au CAD ou sur la feuille } \\
\text { d'administration des médicaments. Certains conditionnements } \\
\text { permettent un nouveau prélèvement dans un produit entamé (liquides) }\end{array}$ & \\
\hline & $\begin{array}{l}\text { 22. Substitution du rebut par un autre médicament - L'intervenant } \\
\text { remplace le médicament contenu dans le conditionnement par un } \\
\text { autre produit avant d'enregistrer le rebut devant témoin }\end{array}$ & \\
\hline \multirow[t]{2}{*}{$\begin{array}{l}\text { V. Utilisation de patients } \\
\text { fictifs }\end{array}$} & $\begin{array}{l}\text { 23. Opération effectuée au nom de patients « fictifs » - Il est possible } \\
\text { de faire des opérations au nom de patients « fictifs » afin d'accéder } \\
\text { rapidement au CAD. }\end{array}$ & $\begin{array}{l}\text { - Tous } \\
\text { - Manually admitted } \\
\text { patient profile* }\end{array}$ \\
\hline & $\begin{array}{l}\text { 24. Admission d'un faux patient - Il est possible d'admettre un patient } \\
\text { qui n'est pas actuellement hospitalisé dans le CAD et de faire des sorties } \\
\text { de médicament à son nom }\end{array}$ & \\
\hline \multirow[t]{2}{*}{ Vl. Bris d'équipements } & $\begin{array}{l}\text { 25. Pochette sécurisée défectueuse - Si le bris d'une pochette sécurisée } \\
\text { est connu, il est possible de retirer le médicament A de cette pochette } \\
\text { lorsqu'on ouvre le tiroir pour y prendre le médicament B dans une autre } \\
\text { pochette. Le nom de l'usager n'est pas associé au médicament détourné, } \\
\text { un écart d'inventaire sera créé lors de l'opération suivante }\end{array}$ & $\begin{array}{l}\text { - Tous } \\
\text { Discrepancy by station* }\end{array}$ \\
\hline & $\begin{array}{l}\text { 26. Tiroir matriciel avec pochettes non sécurisées moins sécuritaire - } \\
\text { Il est possible d'ouvrir le tiroir pour prendre le médicament A et de retirer } \\
\text { en même temps le médicament B. Le nom de la personne ne sera } \\
\text { enregistré que pour la sortie du médicament A }\end{array}$ & \\
\hline $\begin{array}{l}\text { VIII. Rappel de lots de } \\
\text { médicaments }\end{array}$ & $\begin{array}{l}\text { 27. Rappel de lots - L'intervenant retire du CAD les médicaments du ou } \\
\text { des lots concernés par le rappel et en conserve une partie }\end{array}$ & $\begin{array}{l}\text { - Assistant technique en } \\
\text { pharmacie }\end{array}$ \\
\hline
\end{tabular}

\section{RÉSULTATS}

Le tableau 1 présente le profil des mouvements (aussi appelés transactions) et des écarts d'inventaire de substances désignées par CAD du 3 juin au 2 novembre 2015. Durant la période d'étude, 17950 mouvements (117/jour) et 221 écarts d'inventaire $(1,4 /$ jour $)$ ont été observés. Le taux d'écart d'inventaire par mouvement est de $1,2 \%$. Parmi les 221 écarts d'inventaire, 91 (0,6/jour; 41,2\%) sont des écarts complexes. Ces écarts découlent de situations où la quantité réelle que l'usager a sortie était différente de la quantité inscrite à l'écran. Tous les écarts d'inventaire sont résolus, puisque leur justification est obligatoire. Lors de la période étudiée, aucun détournement n’a été observé, tous les écarts étaient justifiés et correspondaient à des erreurs humaines. Concernant la distribution des écarts d'inventaire par unité de soins et par CAD, le nombre de mouvements est corrélé au nombre total d'écarts d'inventaire $\left(R^{2}=0,908\right)$ et au nombre d'écarts complexes $\left(R^{2}=0,946\right)$.

Le tableau 2 présente le profil des écarts d'inventaire par quart de travail des infirmières. La moitié des écarts d'inventaire surviennent entre $10 \mathrm{~h}$ et $16 \mathrm{~h}$. Le nombre d'écarts d'inventaire par quart de travail est corrélé au nombre de mouvements par quart de travail $\left(R^{2}=0,963\right)$. Toutefois, le coefficient de corrélation entre le nombre d'écarts par heure et le nombre de mouvements par heure est moins élevé $\left(R^{2}=0,830\right)$.

Le tableau 3 présente le profil des mouvements et des écarts d'inventaire par substance désignée du 3 juin au 2 novembre 2015. Le nombre d'écarts d'inventaire par substance désignée est corrélé au nombre de mouvements de chaque substance désignée $\left(R^{2}=0,965\right)$. Les taux d'écart d'inventaire par mouvement selon les classes de substances désignées sont nommés par ordre décroissant : 2,7\% pour les drogues contrôlées (barbituriques, méthylphénidate et étomidate); $1,8 \%$ pour les benzodiazépines et $1,1 \%$ pour les narcotiques (opiacés, nabilone et kétamine).

Le tableau 4 présente le profil des modes de défaillance que le panel d'experts et le groupe de discussion ont observés par activité de gestion des CAD. Au total, 27 modes de défaillance concernant sept activités de gestion des CAD ont été constatés. Les usagers concernés et des rapports de surveillance impliqués sont également détaillés. Dans le tableau 4, un médicament est défini comme étant un format ou une unité de distribution aux 
This single copy is for your personal, non-commercial use only.

For permission to reprint multiple copies or to order presentation-ready copies for distribution, contact CJHP at cjhpedit@cshp.ca

\section{Tableau 5. Présentation des six mesures de surveillance des substances désignées dispensées par cabinets automatisés décentralisés (CAD)}

Mise en place

Mesures de surveillance

Mesures déjà en place avant 1. Le personnel de la pharmacie procède à la conciliation en temps réel des écarts dus au personnel la tenue de l'étude soignant et de l'analyse hebdomadaire de la justification des écarts d'inventaire, avec la relance du personnel soignant lorsque les justificatifs sont insatisfaisants.

2. Le personnel de la pharmacie vérifie hebdomadairement l'assignation des doses de substances désignées données à des patients fictifs et non à de véritables patients (c.-à-d. approche non autorisée mais parfois utilisée pour l'administration urgente de médicaments avant que le profil d'un patient ne soit affiché dans un $C A D$ ).

Mesures supplémentaires et complémentaires à mettre en place
3. Le personnel de la pharmacie vérifie mensuellement par échantillonnage la concordance de chaque transaction, dont la prescription, la dispensation, I'administration au patient et la destruction ou le rebut si nécessaire.

4. Le personnel de la pharmacie vérifie mensuellement par échantillonnage la gestion des retours de médicaments par le truchement des CAD.

5. Le personnel de la pharmacie vérifie mensuellement par échantillonnage la concordance entre la quantité dispensée au moyen du logiciel de gestion des réserves de substances désignées (GesPharx) et la quantité réapprovisionnée dans les CAD.

6. Des améliorations ont été apportées au formulaire électronique d'administration des médicaments (FADMe) afin de permettre au personnel soignant de consulter en temps réel les transactions réalisées dans les CAD à partir de ce FADMe (c.-à-d. plus du tiers des lits de l'établissement ont actuellement recours à l'enregistrement électronique des doses administrées). fins d'administration d'une dose de médicament à un patient donné.

Le tableau 5 présente les six mesures de surveillance des substances désignées, dispensées par CAD. Deux mesures de surveillance étaient déjà en place avant le début de l'étude. Quatre mesures de surveillance supplémentaires ont été déterminées à la suite des rencontres avec le panel d'experts et le groupe de discussion.

\section{DISCUSSION}

Il existe relativement peu de données sur l'utilisation optimale des CAD, sur les risques de détournement et sur les mesures de surveillance. Cette étude apporte des données provenant d'un centre hospitalier universitaire canadien.

Pendant la période étudiée, le taux d'écart d'inventaire par mouvement de substances désignées est relativement rassurant (1,2 \% soit 1,4/jour). Quel que soit le paramètre observé (c.-à-d. quart de travail, heure, substance désignée, CAD, écart complexe), le nombre d'écarts d'inventaire est corrélé au nombre de mouvements. Plus il y a d'opérations réalisées au CAD, plus le risque d'écarts d'inventaire est élevé. Le taux élevé d'écart d'inventaire par mouvement de $25,9 \%$ pour le CAD des soins de longue durée 2 s'explique par l'implantation récente du cabinet et par le manque de maîtrise de l'outil par l'équipe infirmière. Dans la presque totalité des cas, les écarts d'inventaire découlent d'erreurs d'inattention ou du nonrespect des politiques et procédures d'utilisation du CAD par le personnel soignant.

L'utilisation des CAD contribue probablement à renforcer le contrôle. La surveillance des écarts d'inventaire par le personnel de la pharmacie permet d'éveiller l'attention sur un éventuel détournement de substances désignées, mais la détection de détournements réels nécessite plusieurs preuves.

Cette étude nous a permis de découvrir 27 modes de défaillance dans le cadre de l'utilisation des CAD. Bien que cette technologie sécurise le circuit du médicament, l'usager bien formé et bien informé peut détecter les failles et contourner la technologie. Les discussions menées avec le panel d'experts et les infirmières démontrent l'importance de déceler ces modes de défaillance afin de réduire les risques au quotidien. La compréhension des failles peut aider à intercepter les intervenants fautifs. Au vu de ces résultats, il semble nécessaire de renforcer nos mesures de surveillance. Ainsi, quatre nouvelles mesures ont été développées et introduites à la suite de cette étude. La surveillance de tous les réapprovisionnements, des retours et des sorties de médicaments des CAD serait excessivement chronophage et irréaliste. Par conséquent, la surveillance de ces paramètres est réalisée mensuellement à l'aide d'échantillons sélectionnés au hasard. Ce type de contrôles aléatoires permet également de dissuader les usagers de détourner des médicaments des CAD. La mise à jour du FADMe permet désormais au personnel soignant de voir toutes les doses de médicaments d'un patient donné sorties du CAD durant les dernières 24 heures, à partir du profil de ce patient, figurant dans le FADMe. Il est ainsi possible de déterminer les sorties non requises pour les soins du patient en question. Les CAD utilisés au CHU Sainte-Justine comportent des dizaines de rapports de surveillance, toutefois, plusieurs d'entre eux ne permettent pas de déterminer facilement les pratiques inhabituelles.

L'équipe de Brenn et coll. a mesuré le nombre de divergences par anesthésiste selon divers paramètres, à l'aide d'un tableau de bord conçu pour corréler des données de sources multiples, ceci afin de détecter les détournements de 
médicaments par les utilisateurs de $\mathrm{CAD}^{13}$. La détection de divergences entre les différents systèmes de gestion des informations en anesthésie, à la pharmacie et des $\mathrm{CAD}$ a permis d'identifier un usager suspect. Le praticien suspecté de détournement avait notamment atteint 35 divergences par mois alors que la moyenne de l'ensemble des praticiens était de 5,7 divergences. À la différence de cette étude, notre approche repose sur six mesures de surveillance distinctes et complémentaires à fréquence variable et un tableau de bord manuscrit. Notre étude ne permet pas de comparer la charge de travail et les coûts inhérents aux deux approches.

Cette étude comporte des forces et des limites. Il s'agit d'une étude originale, qui décrit de façon détaillée et pratique les risques de détournement de substances désignées dans un établissement de santé canadien. Comme les CAD sont utilisés dans de nombreux pays, cette réflexion peut inspirer de nombreux établissements, peu importe les technologies utilisées. En ce qui concerne les limites, il s'agit d'une étude menée dans un centre hospitalier mère-enfant. Bien qu'elle porte sur un centre dont la patientèle est spécifique, nous pensons que le principe d'utilisation des CAD est similaire dans la plupart des établissements, peu importe le type de patients. L'étude ne permet pas de vérifier la capacité de détecter des cas de détournements, mais elle présente les mesures de surveillance mises en place. Il serait intéressant de vérifier si ces mesures permettent de détecter des cas de détournement. Certains pourraient penser que la fréquence d'audits est peut-être insuffisante. Ces fréquences seront réévaluées au fil du temps. Toutefois, l'instauration de contrôles ponctuels et aléatoires de détournements des substances désignées est dissuasive pour le personnel.

\section{CONCLUSION}

Cette étude descriptive met en évidence un taux de 1,2\% d'écart d'inventaire pour les mouvements de substances désignées effectués dans 19 CAD au CHU Sainte-Justine durant une période de cinq mois. Nous avons observé 27 modes de défaillance regroupés en sept activités de gestion des médicaments par CAD. Six mesures de surveillance ont été mises en place afin de prévenir le détournement des substances désignées à partir des CAD.

\section{Références}

1. Groupe de travail sur l'introduction de systèmes automatisés et robotisés utilisés pour la distribution des médicaments dans les établissements de santé au Québec. Les systèmes automatisés et robotisés utilisés pour la distribution des médicaments dans les établissements de santé au Québec. Rapport et recommandations du groupe de travail. Québec $(\mathrm{QC})$ : Ministère de la Santé et des Services sociaux, Direction des communications; 2005. Publié au : http://publications.msss.gouv.qc.ca/acrobat/f/documentation/2005/05-71901.pdf. Consulté le 22 mars 2016.

2. American Society of Health-System Pharmacists. ASHP guidelines on the safe use of automated dispensing devices. Am J Health Syst Pharm. 2010; 67(6):483-90.

3. Brisseau L, Bussières JF, Lebel D, Atkinson S, Robinette L, Fortin S, et al . Utilisation de lignes directrices dans le cadre de l'implantation de cabinets automatisés décentralisés en établissement de santé. Can J Hosp Pharm. 2011;64(2):104-15.

4. Institute for Safe Medication Practice (ISMP) guidance on the interdisciplinary safe use of automated dispensing cabinets. Horsham (PA): Institute for Safe Medication Practice; 2008. Publié au : https://www.ismp.org/Tools/ guidelines/ADC/default.asp. Consulté le 22 mars 2016.

5. Grissinger M. Safeguards for using and designing automated dispensing cabinets. P T. 2012;37(9):490-530.

6. Règlement sur les aliments et les drogues, C.R.C., ch. 870. Publié au : http:// laws-lois.justice.gc.ca/fra/reglements/C.R.C.,_ch._870/. Consulté le 12 avril 2016.

7. Baldisseri MR. Impaired healthcare professional. Crit Care Med. 2007;35 (2 Suppl):S106-16.

8. Epstein RH, Gratch DM, Grunwald Z. Development of a scheduled drug diversion surveillance system based on an analysis of atypical drug transactions. Anesth Analg. 2007;105(4):1053-60.

9. Booth JV, Grossman D, Moore J, Lineberger C, Reynolds JD, Reves JG, et al. Substance abuse among physicians: a survey of academic anesthesiology programs. Anesth Analg. 2002;95(4):1024-30.

10. Office of Applied Studies. Summary of findings from the 2001 National Household Survey on Drug Abuse. Washington (DC): Department of Health and Human Services, Substance Abuse and Mental Health Services Administration; 2002. DHHS publication SMA 01-3549, NHDSA série $\mathrm{H}-14$.

11. Kenna GA, Wood MD. Prevalence of substance use by pharmacists and other health professionals. J Am Pharm Assoc (2003). 2004;44(6):684-93.

12. À propos de CanLII [site web]. Ottawa (ON): Institut canadien d'information juridique; Publié au : https://www.canlii.org/fr/info/a_propos.html. Consulté le 22 mars 2016.

13. Brenn BR, Kim MA, Hilmas E. Development of a computerized monitoring program to identify narcotic diversion in a pediatric anesthesia practice. $A m$ J Health Syst Pharm. 2015;72(16):1365-72.

Sophie Dubois travaille à I'Unité de recherche en pratique pharmaceutique et au Département de pharmacie, Centre hospitalier universitaire Sainte-Justine, Montréal, Québec. Elle est aussi candidate au Pharm. D., Faculté des sciences pharmaceutiques et biologiques de Rennes, Rennes, France.

Emmy Bernier, Pharm. D., M. Sc., travaille à I'Unité de recherche en pratique pharmaceutique et au Département de pharmacie, Centre hospitalier universitaire Sainte-Justine, Montréal (Québec)

Julie Rivard, Pharm. D., M. Sc., travaille à I'Unité de recherche en pratique pharmaceutique et au Département de pharmacie, Centre hospitalier universitaire Sainte-Justine, Montréal (Québec).

Lavina Yu, Pharm. D., M. Sc., travaille à I'Unité de recherche en pratique pharmaceutique et au Département de pharmacie, Centre hospitalier universitaire Sainte-Justine, Montréal (Québec).

Suzanne Atkinson, B. Pharm., M. Sc., travaille à I'Unité de recherche en pratique pharmaceutique et au Département de pharmacie, Centre hospitalier universitaire Sainte-Justine, Montréal (Québec).

Jean-François Bussières, B. Pharm., M. Sc, MBA, FCSHP, travaille à I'Unité de recherche en pratique pharmaceutique et au Département de pharmacie, Centre hospitalier universitaire Sainte-Justine, et au Faculté de pharmacie et des sciences pharmaceutiques, Université de Montréal, Montréal, Québec.

Intérêts concurrents : Aucun déclaré.

\section{Address correspondence to:}

Jean-François Bussières

Département de pharmacie

Centre hospitalier universitaire Sainte-Justine

3175, chemin de la Côte Sainte-Catherine

Montréal QC H3T 1C5

Courriel : jf.bussieres@ssss.gouv.qc.ca

Financement : Aucun reçu. 